\title{
CONDITIONS ON UNIQUENESS OF LIMIT POINT AND COMPLETENESS IN CONE POLYGONAL METRIC SPACES
}

\author{
Sie Evan Setiawan ${ }^{1 *}$, Mahmud Yunus $^{2}$ \\ ${ }^{1,2}$ Institut Teknologi Sepuluh Nopember, Surabaya \\ Email : ${ }^{1}$ evan.setiawan15@mhs.matematika.its.ac.id, ${ }^{2}$ yunusm@matematika.its.ac.id \\ ${ }^{*}$ Corresponding author
}

\begin{abstract}
This paper discusses cone polygonal metric spaces. We analyze some characteristics derived from convergence and Cauchyness of sequences. Our result consists of some conditions on uniqueness of limit point and completeness in cone polygonal metric spaces.
\end{abstract}

Keywords: Cone metric spaces, cone polygonal metric spaces, convergence and Cauchyness, completeness, nested closed-ball property

\section{INTRODUCTION}

The concept of metric spaces is a major subject in mathematical analysis. There had been introductions of generalized metric spaces within various forms. An interesting generalization was found in cone metric spaces [1]. Cone metric space is obtained by substituting the codomain of metric function with a partially ordered Banach space. The order in Banach spaces is defined by a subset called cone, similar to how ordering in the set of real numbers is defined by the set of positive real numbers.

The concept of cone metric spaces was generalized further by replacing the property of triangular inequality with similar inequality which contains four or more elements. The results are cone rectangular metric spaces [2], cone pentagonal metric spaces [3], cone hexagonal metric spaces [4], cone heptagonal metric spaces [5], and so on. However, these spaces share similar characteristics. Therefore, they can be studied as one group, namely cone polygonal metric spaces.

Some basic properties of metric spaces are not present in cone polygonal metric spaces. For example, a convergent sequence can have multiple limit points and is not necessarily Cauchy [6]. This motivates us to further analyze correlation between convergent sequences and Cauchy sequences. We discovered that simultaneous presence of convergence and Cauchyness would lead to uniqueness of limit point.

We also analyze conditions that indicate completeness of a given space. We found sequential compactness to be a sufficient condition for completeness. Other than that, we establish a necessary and sufficient condition by expanding a nested closed-ball theorem [7] from cone metric spaces to cone polygonal metric spaces.

Our result could be applied in other theories that use iterative sequences. For example, our sufficient conditions on completeness could be directly linked to fixed point theorems $[1,2,8$, 
9]. It should be emphasized that we do not use the properties of normal cones in our work. Therefore, our result covers both normal and non-normal cones.

\section{PRELIMINARIES}

We begin by stating the definition of cone and some related terms. Let $E$ be a real Banach space. A subset $P \subset E$ is called cone if: (i) $P$ is a closed non-empty set and $P \neq\left\{0_{E}\right\}$; (ii) For any positive real numbers $k_{1}, k_{2}$, and $x, y \in P$ holds $k_{1} x+k_{2} y \in P$; (iii) $P \cup(-P)=\left\{0_{E}\right\}$ [1]. Cone $P$ defines partial ordering in $E$ as follows: (i) $x \leqslant y$ if $y-x \in P$; (ii) $x \ll y$ if $y-$ $x \in$ int $P$, where int $P$ denotes interior of $P$ [1].

The following lemmas would be useful for later theorems:

Lemma 1 [10] Let $P$ be a cone in $E$ and $u, v, w \in E$, then

(1) If $u \leqslant v$ and $v \ll w$, then $u \ll w$.

(2) If $0_{E} \preccurlyeq u \ll c$ for each $0_{E} \ll c$, then $u=0_{E}$.

(3) If $u \preccurlyeq v+c$ for each $0_{E} \ll c$, then $u \preccurlyeq v$.

Lemma 2 [10] Let $P$ be a cone in $E$, and $\left(a_{n}\right)$ be a sequence in $P$ such that $\lim _{n \rightarrow \infty}\left\|a_{n}\right\|=0$. Then, for any $c \in E$ with $0_{E} \ll c$, there exists $N$ such that $a_{n} \ll c$ for all $n>N$.

Cone polygonal metric space is a generalization of cone metric spaces. It is obtained by replacing the property of triangular inequality with a similar property consisting of $k$ distinct elements. The formal definition is given as follows:

Definition 1 Let $E$ be a Banach space with cone, $X$ be a non-empty set, and $k$ be a natural number greater than three. A function $d: X \times X \rightarrow E$ is called cone $k$-gonal metric iffor every $x, y \in X$ :

(1) $d(x, y) \geqslant 0_{E}$ and $d(x, y)=0_{E}$ if and only if $x=y$

(2) $d(x, y)=d(y, x)$

(3) $d(x, y) \preccurlyeq d\left(x, z_{1}\right)+\sum_{i=1}^{k-3} d\left(z_{i}, z_{i+1}\right)+d\left(z_{k-2}, y\right)$ for all distinct $z_{1}, z_{2}, \ldots, z_{k-2} \in$ $X-\{x, y\}$

The pair $(X, d)$ is then called a cone $k$-gonal metric space.

As in cone metric spaces, we can define convergence and Cauchy-ness of sequences in cone polygonal metric spaces.

Definition 2 Let $(X, d)$ be a cone $k$-gonal metric space, $\left\{x_{n}\right\}$ be a sequence in $X$, and $x \in$ $X .\left\{x_{n}\right\}$ is called a Cauchy sequence if for every $c \in E$ with $0_{E} \ll c$, there exist $N$ such that $d\left(x_{n}, x_{m}\right) \ll c$ for all $m, n>N$. $\left\{x_{n}\right\}$ is called convergent to $x$ if for every $c \in E$ with $0_{E} \ll c$, there exist $N$ such that $d\left(x_{n}, x\right) \ll c$ for all $n>N$.

A cone k-gonal metric space $(X, d)$ is called complete if every Cauchy sequence in $X$ is convergent. If for any sequence in $X$, there exists a convergent subsequence, then $(X, d)$ is called sequentially compact cone k-gonal metric space. 


\section{PROPERTIES IN CONE POLYGONAL METRIC SPACES}

\subsection{Conditions on Uniqueness of Limit Point}

Previous works had found a condition that resulted in the uniqueness of sequences' limit point in cone rectangular metric spaces [11]. While the same condition has been established in cone polygonal metric spaces [9], it was limited to spaces with normal cones only. We expand this condition to cover cone polygonal metric spaces in general (regardless of normality):

Theorem 1 Let $k>3$, and $(X, d)$ be a cone $k$-gonal metric space. If a sequence $\left(x_{n}\right)$ in $X$ is both convergent and Cauchy simultaneously, then limit of $\left(x_{n}\right)$ is unique.

Proof: Let $\left(x_{n}\right)$ be a sequence in $(X, d)$ that is both convergent and Cauchy, and let $x$ and $y$ be limit points of $\left(x_{n}\right)$. For any $c \in E$ with $0_{E} \ll c$, there exist $N$ such that for every distinct $n_{1}, n_{2}, \ldots, n_{k-2}>N$, these inequalities hold:

$$
\begin{aligned}
& d\left(x, x_{n_{1}}\right) \ll \frac{c}{k-1} \\
& d\left(x_{n_{1}}, x_{n_{2}}\right) \ll \frac{c}{k-1} \\
& d\left(x_{n_{(k-3)}}, x_{n_{(k-2)}}\right) \ll \frac{c}{k-1} \\
& d\left(x_{n_{(k-2)}}, y\right) \ll \frac{c}{k-1}
\end{aligned}
$$

These lead us to

$$
d(x, y) \preccurlyeq d\left(x, x_{n_{1}}\right)+\sum_{i=1}^{k-3} d\left(x_{n_{i}}, x_{n_{(i+1)}}\right)+d\left(x_{n_{(k-2)}}, y\right) \ll c
$$

Since $c$ is arbitrary, by Lemma 1 , we have $d(x, y)=0_{E}$ which implies $x=y$.

A direct implication of Theorem 1 is presented in Corollary 1:

Corollary 1 In complete cone polygonal metric spaces, every Cauchy sequence has a unique limit point.

Furthermore, it is found that the convergence condition in Theorem 1 could be loosened by substituting it with the existence of convergent subsequence, as shown in Theorem 2:

Theorem 2 Let $k>3$, and $(X, d)$ be a cone $k$-gonal metric space. If a sequence $\left(x_{n}\right)$ in $X$ is Cauchy and has a convergent subsequence, then $\left(x_{n}\right)$ is convergent and has a unique limit.

Proof: Let $\left(x_{n}\right)$ be a Cauchy sequence in $(X, d),\left(x_{m_{i}}\right)$ be a convergent subsequence of $\left(x_{n}\right)$, and $x$ be the limit point of $\left(x_{m_{i}}\right)$. For any $c \in E$ with $0_{E} \ll c$, there exists $N$ such that for every distinct $n, m_{i_{1}}, m_{i_{2}}, \ldots, m_{i_{k-2}}>N$, these following inequalities hold:

$$
\begin{gathered}
d\left(x_{n}, x_{m_{i_{1}}}\right) \ll \frac{c}{k-1} \\
d\left(x_{m_{i_{1}}}, x_{m_{i_{2}}}\right) \ll \frac{c}{k-1} \\
\vdots \\
d\left(x_{m_{i_{(k-3)}},} x_{m_{i_{(k-2)}}}\right) \ll \frac{c}{k-1}
\end{gathered}
$$


It follows that

$$
d\left(x_{m_{i(k-2)}}, x\right) \ll \frac{c}{k-1}
$$

$$
d\left(x_{n}, x\right) \preccurlyeq d\left(x_{n}, x_{m_{i_{1}}}\right)+\sum_{j=1}^{k-3} d\left(x_{m_{i_{j}}}, x_{m_{i_{(j+1)}}}\right)+d\left(x_{n_{i_{(k-2)}}}, x\right) \ll c
$$

which confirms that $\left(x_{n}\right)$ is indeed convergent to $x$. Since $\left(x_{n}\right)$ is both convergent and Cauchy, by Theorem 1 , limit of $\left(x_{n}\right)$ is unique.

\subsection{Completeness Conditions}

We found a condition that guarantees the completeness of cone polygonal metric spaces as follows:

Theorem 3 Let $k>3$ and $(X, d)$ be a cone $k$-gonal metric space. If $(X, d)$ is sequentially compact, then $(X, d)$ is complete.

Proof: Let $\left(x_{n}\right)$ be an arbitrary Cauchy sequence in $(X, d)$. Since $(X, d)$ is sequentially compact, then $\left(x_{n}\right)$ has a convergent subsequence. By Theorem $2,\left(x_{n}\right)$ is convergent. Therefore, $(X, d)$ is complete.

Theorem 3 implies that finite cone polygonal metric spaces are complete, as stated in the following theorem:

Theorem 4 Let $k>3$ and $(X, d)$ be a cone $k$-gonal metric space. If $X$ is a finite set, then $(X, d)$ is sequentially compact and complete.

Proof: Let $\left(x_{n}\right)$ be an arbitrary sequence in $(X, d)$. Since $X$ is finite, there must be an element $y \in X$ that appears infinite times in $\left(x_{n}\right)$. Pick a subsequence of $\left(x_{n}\right)$ where each element is $y$. This subsequence is obviously convergent to $y$. Therefore, $(X, d)$ is sequentially compact. By Theorem 3 , we conclude that $(X, d)$ is complete.

A previous result had established a nested closed-ball theorem that provides a necessary and sufficient condition for completeness of cone metric spaces [7]. We expand the theorem to cone polygonal metric spaces.

Theorem 5 Let $k>3$ and $(X, d)$ be a cone $k$-gonal metric space. Let $\left(r_{n}\right)$ be an arbitrary sequence in $P \subset E$ such that for every $c \in E$ with $0_{E} \ll c$, there exists $N$ satisfying $r_{n} \ll c$ for every $n>N$. For any sequence $\left(x_{n}\right)$ in $X$, we define closed ball neighborhood $S_{n}=$ $\left\{x \in X \mid d\left(x, x_{n}\right) \preccurlyeq r_{n}\right\} .(X, d)$ is complete if and only if $S_{1} \supseteq S_{2} \supseteq \cdots \supseteq S_{n} \supseteq \cdots$ implies there is a point $y \in \cap_{n=1}^{\infty} S_{n}$.

Proof: Suppose that $(X, d)$ is complete and $S_{1} \supseteq S_{2} \supseteq \cdots \supseteq S_{n} \supseteq \cdots$ holds. For any $n_{1}<n_{2}$, we have $x_{n_{2}} \in S_{n_{2}} \subseteq S_{n_{1}}$, which implies $d\left(x_{n_{1}}, x_{n_{2}}\right) \preccurlyeq r_{n_{1}}$. Therefore, for any $c \in E$ with $0_{E} \ll c$, there is $N$ such that $d\left(x_{n_{1}}, x_{n_{2}}\right) \leqslant r_{n_{1}} \ll c$ for every $n_{2}>n_{1}>N$. This suggests that $\left(x_{n}\right)$ is a Cauchy sequence. Based on the completeness of $(X, d)$, there exists a point $y \in X$ such that $\left(x_{n}\right)$ converges to $y$. Take any $n_{3}$, we will show that $y \in S_{n_{3}}$. Since $\left(x_{n}\right)$ is Cauchy and convergent to $y$, for every $c \in E$ with $0_{E} \ll c$, there is $N$ such that for distinct $m_{1}, m_{2}, \ldots, m_{k-2}>\max \left\{N, n_{3}\right\}$ these inequalities hold: 


$$
\begin{aligned}
& d\left(x_{m_{1}}, x_{m_{2}}\right) \ll \frac{c}{k-2} \\
& d\left(x_{m_{2}}, x_{m_{3}}\right) \ll \frac{c}{k-2} \\
& d\left(x_{m_{(k-3)}}, x_{m_{(k-2)}}\right) \ll \frac{c}{k-2} \\
& d\left(x_{m_{(k-2)}}, y\right) \ll \frac{c}{k-2}
\end{aligned}
$$

Since $m_{1}>n_{3}$, we have $x_{m_{1}} \in S_{m_{1}} \subseteq S_{n_{3}}$ which implies $d\left(x_{n_{3}}, x_{m_{1}}\right) \preccurlyeq r_{n_{3}}$. Therefore,

$$
d\left(x_{n_{3}}, y\right) \preccurlyeq d\left(x_{n_{3}}, x_{m_{1}}\right)+\sum_{i=1}^{k-3} d\left(x_{m_{i}}, x_{m_{(i+1)}}\right)+d\left(x_{m_{(k-2)}}, y\right) \preccurlyeq r_{n_{3}}+c
$$

By Lemma 1 , we have $d\left(x_{n_{3}}, y\right) \preccurlyeq r_{n_{3}}$ which confirms that $y \in S_{n_{3}}$. Since $n_{3}$ is arbitrary, we have $y \in \bigcap_{n=1}^{\infty} S_{n}$.

Conversely, suppose that $S_{1} \supseteq S_{2} \supseteq \cdots \supseteq S_{n} \supseteq \cdots$ implies there is a point $y \in \bigcap_{n=1}^{\infty} S_{n}$, we'll show that $(X, d)$ is complete. Let $\left(x_{n}\right)$ be a Cauchy sequence and pick a fixed $c_{0} \in E$ with $0_{E} \ll c_{0}$. We could take $n_{1}<n_{2}<\cdots<n_{i}<\cdots$ such that $d\left(x_{n}, x_{m}\right) \ll \frac{c_{0}}{(k-1)^{i+1}}$ for every $n, m>n_{i}$. Consider the subsequence $\left(x_{n_{i}}\right)$, and let $S_{i}^{\prime}=\left\{x \in X \mid d\left(x, x_{n_{i}} \preccurlyeq \frac{c_{0}}{(k-1)^{i}}\right)\right\}$. We will show that $S_{i}^{\prime} \supseteq S_{i+1}^{\prime}$ for any $i$. For distinct $n_{(i+1)}, j_{1}, j_{2}, \ldots, j_{k-3}>n_{i}$, we have

$$
\begin{gathered}
d\left(x_{n_{(i+1)},} x_{j_{1}}\right) \ll \frac{c_{0}}{(k-1)^{i+1}} \\
d\left(x_{j_{1}}, x_{j_{2}}\right) \ll \frac{c_{0}}{(k-1)^{i+1}} \\
\vdots \\
d\left(x_{j_{(k-4)}}, x_{j_{(k-3)}}\right) \ll \frac{c_{0}}{(k-1)^{i+1}} \\
d\left(x_{j_{(k-3)}}, x_{n_{i}}\right) \ll \frac{c_{0}}{(k-1)^{i+1}}
\end{gathered}
$$

For any $z \in S_{i+1}^{\prime}$, we have $d\left(z, x_{n_{(i+1)}}\right) \preccurlyeq \frac{c_{0}}{(k-1)^{i+1}}$, which resulted in $d\left(z, x_{n_{i}}\right) \preccurlyeq d\left(z, x_{n_{(i+1)}}\right)+d\left(x_{n_{(i+1)}}, x_{j_{1}}\right)+\sum_{p=1}^{k-4} d\left(x_{j_{p}}, x_{j_{(p+1)}}\right)+d\left(x_{j_{(k-3)}}, x_{n_{i}}\right) \preccurlyeq \frac{c_{0}}{(k-1)^{i}}$ which implies $S_{i}^{\prime} \supseteq S_{i+1}^{\prime}$. Therefore, there is a point $y \in \bigcap_{i=1}^{\infty} S_{i}^{\prime}$. Since $y \in S_{i}^{\prime}$ for all $i \in \mathbb{N}$, we have $d\left(y, x_{n_{i}}\right) \preccurlyeq \frac{c_{0}}{(k-1)^{i}}$. Observing that $\lim _{i \rightarrow \infty}\left\|\frac{c_{0}}{(k-1)^{i}}\right\|=\lim _{i \rightarrow \infty} \frac{1}{(k-1)^{i}}\left\|c_{0}\right\|=0$, by Lemma 2 we have that for every $c \in E$ with $0_{E} \ll c$, there is $N$ such that

$$
d\left(y, x_{n_{i}}\right) \preccurlyeq \frac{c_{0}}{(k-1)^{i}} \ll c
$$

for every $n_{i}>N$. Thus, $\left(x_{n_{i}}\right)$ is convergent to $y$. By Theorem 2, $\left(x_{n}\right)$ is convergent to $y$. We conclude that $(X, d)$ is complete.

\section{CONCLUSION}

We have analyzed some properties in cone polygonal metric spaces. We showed that in cone polygonal metric spaces, a Cauchy sequence that is convergent or has a convergent 
subsequence must have a unique limit. We proved that a sequentially compact cone polygonal metric space must be complete, thus all finite cone polygonal metric spaces are complete. Finally, we established a necessary and sufficient condition of completeness in cone polygonal metric spaces by using nested-closed ball property.

\section{REFERENCES}

[1] L.-G. Huang and X. Zhang, "Cone Metric Spaces and Fixed Point Theorems of Contractive Mappings," Journal of Mathematical Analysis and Applications, vol. 332, pp. 1468-1476, 2007.

[2] A. Azam, M. Arshad and I. Beg, "Banach Contraction Principle on Cone Rectangular Metric Spaces," Applicable Analysis and Discrete Mathematics, vol. 3, pp. 236-241, 2009.

[3] M. Garg and S. Agarwal, "Banach Contraction Principle on Cone Pentagonal Metric Space," Journal of Advanced Studies in Topology, vol. 3, no. 1, pp. 12-18, 2012.

[4] M. Garg, "Banach Contraction Principle on Cone Hexagonal Metric Space," Ultra Scientist, vol. 26, no. 1, pp. 97-103, 2014.

[5] C. B. Ampadu, "Chatterjea Contraction Mapping Theorem in Cone Heptagonal Metric Space," Fundamental Journal of Mathematics and Mathematical Sciences, vol. 7, no. 1, pp. 15-23, 2017.

[6] B. Samet, "Discussion on "A Fixed Point Theorem of Banach-Caccioppoli Type on a Class of Generalized Metric Spaces" by A. Branciari," Publ. Math. Debrecen, vol. 76, no. 4, pp. 493-494, 2010.

[7] H.-P. Huang and S.-Y. Xu, "Some New Topological Properties in Cone Metric Spaces," J. of Math. (PRC), vol. 35, no. 3, pp. 513-518, 2015.

[8] M. A. Khamsi, "Remarks on Cone Metric Spaces and Fixed Point Theorems of Contractive Mappings," Fixed Point Theory and Applications, 2010.

[9] I. G. Araus and J. L. Ramirez, "Fixed Point Theorems in N-Polygonal Cone Metric Spaces," Bulletin of Mathematical Analysis and Applications, vol. 5, no. 1, pp. 22-35, 2013.

[10] S. Jankovic, Z. Kadelburg and S. Radenovic, "On Cone Metric Spaces: A Survey," Nonlinear Analysis, vol. 74, pp. 2591-2601, 2011.

[11] S. E. Setiawan, Sunarsini and Sadjidon, "Completeness and Fixed Point Theorem in Cone Rectangular Metric Spaces," Journal of Physics: Conference Series, vol. 1490, 2020. 